\title{
Incentive Contract Design for Supply Chain Enterprise's Pollution Abatement with Carbon Tax
}

\author{
Jing Yu $\mathbb{D}^{\mathbb{D}},{ }^{1}$ Chi Zhou $\mathbb{D}^{1},{ }^{1}$ Yixin Wang $\mathbb{D},{ }^{1}$ and Zhibing Liu $\mathbb{D}^{2}$ \\ ${ }^{1}$ School of Management, Tianjin University of Technology, Tianjin 300384, China \\ ${ }^{2}$ Institute of Uncertain Systems, Huanggang Normal University, Hubei 438000, China \\ Correspondence should be addressed to Chi Zhou; czhou@tjut.edu.cn
}

Received 20 February 2021; Revised 5 April 2021; Accepted 19 April 2021; Published 29 April 2021

Academic Editor: Aijun Liu

Copyright ( 2021 Jing Yu et al. This is an open access article distributed under the Creative Commons Attribution License, which permits unrestricted use, distribution, and reproduction in any medium, provided the original work is properly cited.

\begin{abstract}
This paper applies mechanism design to the supply chain enterprise's pollution abatement problem with carbon tax. To maximize the government's expected utility, an uncertain contract model is presented in the framework of principal-agent theory, where the government's assessment of the supply chain enterprise's carbon emission level is described as an uncertain variable. Afterwards, the equivalent model is provided to obtain the optimal contract for the uncertain pollution abatement problem. The results demonstrate that the supply chain enterprise's optimal output decreases with the carbon emission level. Furthermore, the government's optimal transfer payment decreases with the carbon emission level if the carbon tax is low. In contrast, if the carbon tax is high, the optimal transfer payment increases with the carbon emission level. In addition, an increase in the carbon emission level decreases the optimal utilities of both the government and the supply chain enterprise and also leads to the supply chain enterprise's incremental marginal utility. Finally, we provide a numerical example, which illustrates the effectiveness and practicability of the proposed model.
\end{abstract}

\section{Introduction}

The implementation of an environmental governance policy on government agendas around the world has stirred a renewed interest in the optimal mechanism design of pollution abatement. Environmental pollution is caused by the excessive discharge of various industrial pollutants, such as carbon emissions, leading to climate change, air pollution, and water pollution. According to the Global Carbon Budget 2020, the Global Carbon Project's researchers estimate that global carbon emissions in 2019 will increase by $2 \%(+0.8 \%$ to $+3.0 \%$ ) after three years of almost no growth, reaching a new high of $9.9 \pm 0.5 \mathrm{GtC}$. From the report of Climate News Network 2020, almost one-fifth of all the world's carbon emissions come from the supply chain enterprises. According to a McKinsey report on consumer packaged goods (CPG) supply chain enterprises, more than $80 \%$ of the overall emissions come from getting a product from the source to the consumer as part of the supply chain. Mitigating environmental pollution will require the government to adopt substantial abatement mechanisms of industrial pollutants or carbon emissions that can be implemented most cost-effectively by the carbon tax policy [1]. The government's choice of appropriate pollution abatement mechanisms is essential for minimizing the environmental pollution and stimulating the supply chain enterprises to increase productivity, which will further promote economic growth [2]. This paper presents an optimal incentive contract design for an uncertain pollution abatement problem with carbon tax.

The government, as an environmental regulator, usually lacks perfect information on supply chain enterprises' demand for pollution. Because of the influence of such asymmetric information, supply chain enterprises have incentives to misreport their pollution demand if the pollution abatement mechanism is based on such reports. Therefore, many authors (e.g., Kim and Chang [3], Duggan and Roberts [4], and Montero [5]) have designed incentive mechanisms that can induce regulated supply chain enterprises to truthfully reveal their demand. For example, these studies 
present mechanisms whereby the regulator provides the supply chain enterprise a menu of contracts; faced with this menu, the supply chain enterprise's dominant strategy is to truthfully report its private information. By applying principal-agent theory, the relationship between the economy and the environment is discussed under asymmetric carbon emission information. However, few studies in the existing literature investigate how the incentive contract is designed in the uncertain pollution abatement problem when carbon tax is considered.

As a representative approach to mitigating environmental pollution, carbon tax discourages the use of fossil fuels by making carbon emissions more costly [6]. Moreover, this policy also facilitates reductions in carbon emissions via fuel choices or technological innovations. In 1990, a carbon tax policy was first introduced in Finland and was subsequently extended to several other countries. For instance, Japan introduced a carbon tax policy in 2012. The use of carbon taxes has shown broadly positive effects in reducing carbon emissions while generating slightly negative impacts on economic growth. In China, an environmental protection tax law was implemented on 1 January 2018. The carbon tax policy has received increasing attention since the launch of the national carbon trading market. Nevertheless, there are few theoretical studies of the impact of carbon emissions and taxes on the optimal incentive contract design.

To study this problem, we construct an optimal contract model in which the government is regarded as the principal and the supply chain enterprise is regarded as the agent. To this end, an uncertain contract model is presented to maximize the expected utility of the government, in which the government's assessment of the supply chain enterprise's carbon emission level is subjective and is described as an uncertain variable. Then, we provide the crisp equivalent model and obtain the optimal solution of this problem. If the government has historical data on the supply chain enterprise's carbon emissions, then the uncertain information about carbon emission level may be rationally described as a random variable. A model can be built to maximize the government expected net profit, and its equivalent deterministic model can be also obtained naturally by applying the probability measure. Meanwhile, the optimal solution of the contract model under stochastic environment can be also obtained. However, in most practical scenarios, historical data are unavailable. The supply chain enterprise's carbon emission level is often difficult to measure directly. In fact, for a supply chain enterprise without historical data on the carbon emission level, the government can ask domain experts to subjectively evaluate the degree of belief about the supply chain enterprise's carbon emissions. Subsequently, the uncertainty distribution of carbon emissions could be estimated by using uncertainty theory.

The main findings of our paper are as follows. First, if a supply chain enterprise's carbon emission level increases, then the supply chain enterprise is less willing to produce output; that is, the optimal output of the enterprise decreases with the carbon emission level. Second, the government's optimal transfer payment decreases with the carbon emission level if the carbon tax is below a certain threshold. In contrast, if the carbon tax is above this threshold, the government's optimal transfer payment to the supply chain enterprise increases with the carbon emission level. Finally, the optimal utilities of both the government and the enterprise decrease with the carbon emission level.

The remainder of this study is organized as follows. Section 2 reviews the related literature. We present an uncertain contract model in Section 3. Section 4 derives an equivalent model for uncertain pollution abatement problem. Moreover, we obtain the optimal contract for the equivalent model in Section 5. In Section 6, we provide a numerical example to illustrate the effectiveness and practicability of the proposed model. Finally, Section 7 summarizes the main conclusions of this study.

\section{Literature Review}

This study is mainly related to three streams of literature. The first stream concentrates on the economics of the pollution abatement problem. The second stream explores principalagent problems associated with pollution abatement. The last research stream addresses the application of uncertainty theory to principal-agent problems.

In the first stream of literature on the economics of the pollution abatement problem, the effect of government policy on the economics of pollution abatement has been investigated by many researchers [7, 8]. Laffont and Tirole [9] study how spot and futures markets for tradeable pollution permits affect the polluters' compliance decisions. The conclusions can be applied to a variety of situations, such as public transportation, demand-side management, bypass in telecommunications, or forward sales by a private monopolist. Moreover, the researchers discuss the negative impact of plain pollution allowance markets on the environmental pollution innovation. Lothe and Myrtveit [10] establish a formal model to interpret the issues that arise in the multitask environmental problem of implementing an optimal strategy. In recent years, research and practice in the area of carbon tax policy have continued to grow. For instance, Martin et al. [2] examine the effects of carbon tax on manufacturing plants by using the panel data from the UK production census. Liu et al. [6] summarize an analysis of choice preferences to design the carbon tax policy from the viewpoint of Chinese businesses. Fahimnia et al. [11] establish a supply chain optimization model that combines carbon emissions and economic objectives under the scheme of carbon tax policy. In addition, Klenert and Mattauch [12] study the distributional effects of carbon tax reform while considering that households must consume carbonintensive goods in the market. In contrast to these papers, we consider the optimal mechanism design in the pollution abatement problem with carbon tax and analyze how carbon tax affects the optimal contract.

This paper also examines numerous studies of principalagent problems associated with pollution abatement [13]. Helm and Wirl [14] discuss contracting of a principal with an agent if multilateral externalities are present; the example is that of an international climate agreement given private information about the willingness to pay for emissions abatement. By 
studying a hierarchical model of environmental regulation and enforcement, Arguedas and Rousseau [15] investigate the national regulator and the monitoring decision made by a local enforcement agency. In addition, Shrestha [16] designs an incentive mechanism in which the regulator provides a menu of linear price-quantity contracts to each firm. Lika et al. [17] study incentive water pricing schemes under asymmetric information by using a principal-agent model. By applying the agency theory and drawing on the organizational culture, Dubey et al. [18] study a theoretical model of reconfigurable manufacturing systems to integrate the top management's beliefs, participation, and environmental performance. However, our work differs from the studies cited above in three aspects. First, the carbon emission level is the private information of the supply chain enterprise. Second, we consider a carbon tax that the supply chain enterprise pays to the government in the pollution abatement problem. Third, we assume that the government's subjective assessment of the supply chain enterprise's carbon emission level is described as an uncertain variable.

The last stream of literature examines the application of uncertainty theory to principal-agent problems. The existing literature characterizes the uncertain information in principal-agent problems as a random variable or a fuzzy variable $[19,20]$. However, due to the influence of subjective factors and the lack of historical data, characterizing the information uncertainty as randomness is not entirely reasonable. Therefore, uncertainty theory, an axiomatic approach based on the subjective information, has been proposed, including an uncertain variable, uncertainty distribution, and expected value [21]. Since then, uncertainty theory has attracted considerable attention among researchers in related fields as an important mathematical approach to dealing with information uncertainty. Many researchers apply uncertainty theory to principal-agent problems [22]. For instance, $\mathrm{Mu}$ et al. [23] study a principal-agent problem between one enterprise and one rural migrant worker and then establish an uncertain contract model. Zhou et al. [24] establish an uncertain model of principal-agent problem under loss aversion and inequity aversion and analyze how loss aversion and inequity aversion affect the wage structure in optimal contract design. By studying an uncertain principal-agent model, Zhou et al. [25] investigate the effect of referral services on the optimal contract with CPC or CPS payments. In addition, the application of uncertainty theory in supply chain management has been extensively researched in the literature [26, 27]. Similar to the above literature, our work depicts the government's subjective assessment of the supply chain enterprise's carbon emission level by an uncertain variable.

\section{Uncertain Pollution Abatement Model}

Consider an uncertain pollution abatement problem with two participants: the government (she) and the supply chain enterprise (he). The government is the principal, and the supply chain enterprise is the agent. To induce the supply chain enterprise to truthfully reveal the carbon emission level, the government should design a mechanism to optimize the trade-off between the economic development and environmental protection.
The carbon emission level is the private information of the supply chain enterprise, and the government cannot observe it exactly. The government's subjective assessment of the supply chain enterprise's carbon emission level can be characterized as an uncertain variable $\xi$ in the range of $[a, b]$, where $0 \leq a<b<+\infty$. The uncertain variable $\xi$ has the uncertainty distribution function $\Phi(x)$; it is assumed that $\phi(x)$ is the derivative of $\Phi(x)$, where $\Phi(x)$ and $\phi(x)$ satisfy $(\Phi(x) / \phi(x)) / \mathrm{d} x \geq 0, \forall x \in[a, b]$. Let $q(x)$ be the output of the supply chain enterprise, where $x$ denotes the carbon emissions he realizes in the production process. The degree of pollution of the environment is $p(x)$, which can be practically measured by air quality, water quality, and biological pollution. $C(q(x), x)$ denotes the supply chain enterprise's production cost at a given level of carbon emissions. Moreover, the transfer payment the government makes to the supply chain enterprise is denoted by $t(x)$, and $G(q(x))$ represents the revenue the government obtains if the supply chain enterprise's output is $q(x)$. In addition, $\beta x$ is the corresponding carbon tax the supply chain enterprise pays to the government, where $\beta$ represents the tax rate of the carbon emissions quota. This assumption implies that an increase in carbon emissions increases the carbon tax if the tax rate is unchanged. Thus, the contract that the government designed can be characterized by a mechanism $(q(\cdot), t(\cdot))$.

In the pollution abatement problem, an uncertain contract model is presented to maximize the expected utility of government. The government could induce the supply chain enterprise to truthfully reveal carbon emission level by the optimal incentive contract. To be more specific, several assumptions are listed as follows.

(3.1) For the supply chain enterprise's production cost function $C(q, x)$, we assume that

$$
\begin{aligned}
& \frac{\partial C(q, x)}{\partial q}>0 \\
& \frac{\partial C(q, x)}{\partial x}>0 .
\end{aligned}
$$

That is, when the carbon emission the supply chain enterprise realizes is constant, the supply chain enterprise's production cost increases as the output $q$ increases; and when the output is constant, the supply chain enterprise's production cost increases as the realized carbon emission level increases. In addition, the cost function also satisfies the following conditions:

$$
\begin{aligned}
& \frac{\partial^{2} C(q, x)}{\partial q^{2}}>0, \\
& \frac{\partial^{2} C(q, x)}{\partial q \partial x}>0, \\
& \frac{\partial^{3} C(q, x)}{\partial q \partial x^{2}} \geq 0, \\
& \frac{\partial^{3} C(q, x)}{\partial q^{2} \partial x} \geq 0 .
\end{aligned}
$$


Referring to some literature $[8,13,16]$, we also assume that an increase in the supply chain enterprise's output and carbon emission level will increase the production cost. This assumption means that the supply chain enterprise's output is positively correlated with the production cost due to the law of diminishing marginal profit. Moreover, the enterprise with higher carbon emission level may have a higher production quantity and lower green production technology. An efficient production output and advanced green production technology can help the supply chain enterprise reduce production costs.

(3.2) The degree of pollution $p(x)$ to the environment is increasing in the realized carbon emission level $x$; that is,

$$
\frac{\mathrm{d} p(x)}{\mathrm{d} x}>0, \quad 0<p(x)<p_{0}, \forall x \in[a, b],
$$

where $p_{0}$ is constant, implying the highest degree of environmental pollution. Clearly, this means that there is an upper bound on the degree of environmental pollution.

(3.3) The transfer payment $t(x)$ of the government and the output $q(x)$ of the supply chain enterprise are almost everywhere continuously differentiable, and

$$
0 \leq t(x) \leq T, \quad \forall x \in[a, b] .
$$

(3.4) The government's revenue function $G(q)$ is an increasing and concave function with the output $q$; that is,

$$
\begin{gathered}
\frac{\mathrm{d} G(q)}{\mathrm{d} q}>0, \\
\frac{\mathrm{d}^{2} G(q)}{\mathrm{d} q^{2}} \leq 0, \\
G(0)=0 .
\end{gathered}
$$

(3.5) For each contract $(q(x), t(x))$, we assume that

$$
\frac{\mathrm{d} G(q)}{\mathrm{d} q}-\frac{\partial C(q, x)}{\partial q} \geq 0, \quad \forall x \in[a, b] .
$$

The government's utility function can be represented by

$$
\begin{aligned}
V(q(x), t(x), x)= & \lambda(G(q(x))-t(x)+\beta x) \\
& +(1-\lambda)\left(p_{0}-p(x)\right),
\end{aligned}
$$

where $\lambda \in(0,1)$ represents the government's preferences for environment. If $\lambda$ is close to 0 , the government is only concerned with the environment. If $\lambda$ is close to 1 , the government behaves as a pure expected utility-maximizing entity concerned only with the economy. On the right-hand side of equation (7), the first term represents the sum of the revenue and net carbon tax of the transfer payment. The second term also implies the change value of the initial state and the pollution state of the environment. The expected utility of the government can be obtained as follows:

$$
\begin{aligned}
E[V(q(\xi), t(\xi), \xi)]= & E[\lambda(G(q(\xi))-t(\xi)+\beta \xi) \\
& \left.+(1-\lambda)\left(p_{0}-p(\xi)\right)\right] .
\end{aligned}
$$

Since the government cannot exactly assess the carbon emission level of the supply chain enterprise, the carbon emission level cannot be observed. To motivate the supply chain enterprise to truthfully reveal their private information, the incentive-compatible constraint of the supply chain enterprise should be written as follows:

$t(x)-\beta x-C(q(x), x) \geq t(y)-\beta y-C(q(y), x), \quad \forall x, y \in[a, b]$.

In addition, the supply chain enterprise has two choices: one is to accept the contract that the government designed; alternatively, the supply chain enterprise can reject the contract. Only if the difference between the transfer payment and the carbon tax is larger than the production cost is it rational for the enterprise to participate in production; that is,

$U(q(x), t(x), x)=t(x)-\beta x-C(q(x), x) \geq 0, \quad \forall x \in[a, b]$,

which represents the participation constraint of the uncertain contract model.

The uncertain contract model of pollution abatement problem can be written as follows:

$$
\begin{array}{ll}
\max _{\{q(\cdot), t(\cdot)\}} E[V(q(\xi), t(\xi), \xi)] \\
\text { s.t. } \quad t(x)-\beta x-C(q(x), x) \geq t(y)-\beta y-C(q(y), x), \quad \forall x, y \in[a, b], \\
\quad t(x)-\beta x-C(q(x), x) \geq 0, \quad \forall x \in[a, b] .
\end{array}
$$




\section{Equivalent Model for the Uncertain Pollution Abatement Problem}

In this section, the equivalent form of the uncertain pollution abatement model is considered to obtain the optimal solution of the model (11). We first consider the incentive compatibility constraint (9) and then derive the following proposition.

Proposition 1. For any $x \in[a, b]$, the incentive compatibility constraint (9) is equivalent to

$$
\left\{\begin{array}{l}
\frac{\mathrm{d} t(x)}{\mathrm{d} x}=\beta+\frac{\partial C(q, x)}{\partial q} \cdot \frac{\mathrm{d} q(x)}{\mathrm{d} x} \\
\frac{\mathrm{d} q(x)}{\mathrm{d} x}<0 .
\end{array}\right.
$$

Proof. Let $R(x, y)=t(y)-\beta y-C(q(y), x)$, which represents the utility of the supply chain enterprise obtains with the carbon emission level $x$ but choosing the mechanism $(q(\cdot), t(\cdot))$, where $x \neq y$. Therefore, the incentive compatibility constraint (9) is rewritten as

$$
R(x, x) \geq R(x, y), \quad \forall x, y \in[a, b] .
$$

For any $x \in[a, b]$, from the first-order condition

$$
\left.\frac{\partial R(x, y)}{\partial y}\right|_{y=x}=0
$$

we obtain that

$$
\frac{\mathrm{d} t(x)}{\mathrm{d} x}=\beta+\frac{\partial C(q(x), x)}{\partial q} \cdot \frac{\mathrm{d} q(x)}{\mathrm{d} x}, \quad \forall x \in[a, b] .
$$

Moreover, the second-order condition should also be satisfied; that is,

$$
\left.\frac{\partial^{2} R(x, y)}{\partial y^{2}}\right|_{y=x}<0
$$

Furthermore, we derive

$$
\frac{\mathrm{d}^{2} t(x)}{\mathrm{d} x^{2}}-\frac{\partial^{2} C(q(x), x)}{\partial q^{2}} \cdot\left(\frac{\mathrm{d} q(x)}{\mathrm{d} x}\right)^{2}-\frac{\partial C(q(x), x)}{\partial q} \cdot \frac{\mathrm{d}^{2} q(x)}{\mathrm{d} x^{2}}<0 .
$$

By differentiating equation (15) with respect to $x$, we obtain that

$$
\begin{aligned}
\frac{\mathrm{d}^{2} t(x)}{\mathrm{d} x^{2}}= & \frac{\partial^{2} C(q(x), x)}{\partial q^{2}}\left(\frac{\mathrm{d} q(x)}{\mathrm{d} x}\right)^{2}+\frac{\partial^{2} C(q(x), x)}{\partial q \partial x} \frac{\mathrm{d} q(x)}{\mathrm{d} x} \\
& +\frac{\partial C(q(x), x)}{\partial q} \frac{\mathrm{d}^{2} q(x)}{\mathrm{d} x^{2}} .
\end{aligned}
$$

Substituting equation (18) into (17) yields that

$$
\frac{\partial^{2} C(q(x), x)}{\partial q \partial x} \frac{\mathrm{d} q(x)}{\mathrm{d} x}<0, \quad \forall x \in[a, b] .
$$

Since we have $\left(\partial^{2} C(q, x) / \partial q \partial x\right)>0$ by assumption (3.1), it follows that $(\mathrm{d} q(x) / \mathrm{d} x)<0$. In addition, equation (15) can be rewritten as

$$
\frac{\mathrm{d} t(x)}{\mathrm{d} x}=\beta+\frac{\partial C(q, x)}{\partial q} \cdot \frac{\mathrm{d} q(x)}{\mathrm{d} x} .
$$

In addition, when $y>x$,

$$
\begin{aligned}
t(y)-\beta y-(t(x)-\beta x) & =\int_{x}^{y} \frac{\partial C(q(\tau), \tau)}{\partial q} \frac{\mathrm{d} q(\tau)}{\mathrm{d} \tau} \mathrm{d} \tau \\
& \leq \int_{x}^{y} \frac{\partial C(q(\tau), x)}{\partial q} \frac{\mathrm{d} q(\tau)}{\mathrm{d} \tau} \mathrm{d} \tau \\
& =C(q(y), x)-C(q(x), x),
\end{aligned}
$$

from $\left(\partial^{2} C(q, x) / \partial q \partial x\right)>0$ and $(\mathrm{d} q(x) / \mathrm{d} x)<0$.

When $y<x$, inequality (21) also holds. Therefore, the inequality $t(x)-\beta x-C(q(x), x) \geq t(y)-\beta y-C(q(y), x)$ holds for any $x, y \in[a, b]$.

Proposition 1 implies that as the carbon emission level $x$ increases, the regulated polluting supply chain enterprise lowers his output $q(x)$. This observation implies that the optimal output of the regulated polluting supply chain enterprise will decrease with the increase in the carbon emission level.

Next, we discuss the participation constraint (10) and derive the following proposition.

Proposition 2. The participation constraint (10) is equivalent to

$$
U(q(b), t(b), b)=0 \text {. }
$$

Proof. Differentiating equation (10) with respect to $x$ yields $\frac{\mathrm{d} U(q(x), t(x), x)}{\mathrm{d} x}=\frac{\mathrm{d} t(x)}{\mathrm{d} x}-\beta-\frac{\partial C(q(x), x)}{\partial q} \cdot \frac{\mathrm{d} q(x)}{\mathrm{d} x}-\frac{\partial C(q(x), x)}{\partial x}$.

From equation (15) and assumption (3.1), we obtain

$$
\frac{\mathrm{d} U(q(x), t(x), x)}{\mathrm{d} x}=-\frac{\partial C(q(x), x)}{\partial x}<0,
$$

which shows that the supply chain enterprise's utility function is decreasing in the carbon emission level; that is, for any $x \in[a, b]$, there exists

$$
U(q(x), t(x), x)>U(q(b), t(b), b) \geq 0 .
$$

Therefore, provided that $U(q(b), t(b), b)=0$, the participation constraint can be satisfied.

Finally, we further investigate the objective function of the government and obtain the equivalent form as follows. 
Proposition 3. The objective function of the government can be rewritten as

$$
\begin{aligned}
& E[V(q(\xi), t(\xi), \xi)] \\
& =\int_{a}^{b}\left[\lambda(G(q(x))-t(x)+\beta x)+(1-\lambda)\left(p_{0}-p(x)\right)\right] \phi(x) \mathrm{d} x .
\end{aligned}
$$

Proof. Differentiating $V(q(\xi), t(\xi), \xi)$ with respect to $x$ yields

$$
\begin{aligned}
\frac{\mathrm{d} V(q(x), t(x), x)}{\mathrm{d} x} & =\lambda\left[\frac{\mathrm{d} G(q(x))}{\mathrm{d} q} \cdot \frac{\mathrm{d} q(x)}{\mathrm{d} x}-\frac{\mathrm{d} t(x)}{\mathrm{d} x}+\beta\right]-(1-\lambda) \frac{\mathrm{d} p(x)}{\mathrm{d} x} \\
& =\lambda\left[\frac{\mathrm{d} G(q(x))}{\mathrm{d} q}-\frac{C}{\partial q}\right] \cdot \frac{\mathrm{d} q(x)}{\mathrm{d} x}-(1-\lambda) \frac{\mathrm{d} p(x)}{\mathrm{d} x}
\end{aligned}
$$
obtain

By assumptions (3.2) and (3.5) and Proposition 1, we

$$
\left\{\begin{array}{l}
\frac{\mathrm{d} G(q(x))}{\mathrm{d} q}-\frac{\partial C(q(x), x)}{\partial q} \geq 0 \\
\frac{\mathrm{d} p(x)}{\mathrm{d} x}>0 \\
\frac{\mathrm{d} q(x)}{\mathrm{d} x}<0
\end{array}\right.
$$

$$
\begin{array}{ll}
\text { s.t. } & \frac{\mathrm{d} q(x)}{\mathrm{d} x}<0, \quad \forall x \in[a, b], \\
& t(b)-\beta b-C(q(b), b)=0 .
\end{array}
$$$$
\max _{\{q(\cdot), t(\cdot)\}} \int_{a}^{b}\left[\lambda(G(q(x))-t(x)+\beta x)+(1-\lambda)\left(p_{0}-p(x)\right)\right] \phi(x) \mathrm{d} x
$$$$
\frac{\mathrm{d} t(x)}{\mathrm{d} x}=\beta+\frac{\partial C(q, x)}{\partial q} \cdot \frac{\mathrm{d} q(x)}{\mathrm{d} x}, \quad \forall x \in[a, b],
$$

\section{Optimal Solution of the Equivalent Model}

This section obtains the optimal solution of the equivalent contract model. The following theorem states the main result that there exists an optimal contract of the equivalent model of the uncertain pollution abatement problem.

Theorem 1. If $\left(q^{*}(x), t^{*}(x)\right)$ is the optimal contract of model (30), then we have derive that that is, $(\mathrm{d} V(q(x), t(x), x) / \mathrm{d} x)<0$. It implies that the government's utility function decreases with the carbon emission level $x$.

With reference to Liu and Ha [28], the government's expected utility function is presented as

$$
\begin{aligned}
& E[V(q(\xi), t(\xi), \xi)] \\
& =\int_{a}^{b}\left[\lambda(G(q(x))-t(x)+\beta x)+(1-\lambda)\left(p_{0}-p(x)\right)\right] \phi(x) \mathrm{d} x .
\end{aligned}
$$

According to Propositions 1-3, it is clear to prove that model (11) is equivalent to

$$
\begin{gathered}
\frac{\mathrm{d} G\left(q^{*}(x)\right)}{\mathrm{d} q}-\frac{\partial C\left(q^{*}(x), x\right)}{\partial q}-\frac{\partial^{2} C\left(q^{*}(x), x\right)}{\partial x \partial q} \cdot \frac{\Phi(x)}{\phi(x)}=0 \\
t^{*}(x)=\beta x+C\left(q^{*}(x), x\right)+\int_{x}^{b} \frac{\partial C\left(q^{*}(\tau), \tau\right)}{\partial \tau} \mathrm{d} \tau
\end{gathered}
$$

Proof. From $U(q(b), t(b), b)=0$ and equation (24), we 


$$
U(q(x), t(x), x)=\int_{x}^{b} \frac{\partial C(q(\tau), \tau)}{\partial \tau} \mathrm{d} \tau
$$

Furthermore, by equation (10), we have

$$
t(x)-\beta x=C(q(x), x)+\int_{x}^{b} \frac{\partial C(q(\tau), \tau)}{\partial \tau} \mathrm{d} \tau .
$$

Let $S=\int_{a}^{b}(G(q(x))-t(x)+\beta x) \phi(x) \mathrm{d} x$, and then it follows from equation (34) that

$$
S=\int_{a}^{b}\left(G(q(x))-C(q(x), x)-\int_{x}^{b} \frac{\partial C(q(\tau), \tau)}{\partial \tau} \mathrm{d} \tau\right) \phi(x) \mathrm{d} x .
$$

Define $Y=\int_{a}^{b} \int_{x}^{b}(\partial C(q(\tau), \tau) / \partial \tau) \mathrm{d} \tau \phi(x) \mathrm{d} x$, and then

$$
S=\int_{a}^{b}(G(q(x))-C(q(x), x)) \phi(x) \mathrm{d} x-Y .
$$

Since

$$
\begin{aligned}
Y & =\int_{a}^{b}\left(\int_{x}^{b} \frac{\partial C(q(\tau), \tau)}{\partial \tau} \mathrm{d} \tau\right) \phi(x) \mathrm{d} x \\
& =\int_{a}^{b}\left(\int_{x}^{b} \frac{\partial C(q(\tau), \tau)}{\partial \tau} \mathrm{d} \tau\right) \mathrm{d} \Phi(x) \\
& =\int_{a}^{b} \Phi(x) \frac{\partial C(q(x), x)}{\partial x} \mathrm{~d} x-\Phi(a) \int_{a}^{b} \frac{\partial C(q(\tau), \tau)}{\partial \tau} \mathrm{d} \tau \\
& =\int_{a}^{b} \Phi(x) \frac{\partial C(q(x), x)}{\partial x} \mathrm{~d} x,
\end{aligned}
$$

$$
\begin{aligned}
S & =\int_{a}^{b}(G(q(x))-C(q(x), x)) \phi(x) \mathrm{d} x-\int_{a}^{b} \Phi(x) \frac{\partial C(q(x), x)}{\partial x} \mathrm{~d} x \\
& =\int_{a}^{b}\left\{(G(q(x))-C(q(x), x)) \phi(x)-\Phi(x) \frac{\partial C(q(x), x)}{\partial x}\right\} \mathrm{d} x \quad\left[(G(q(x))-C(q(x), x))-\frac{\partial C(q(x), x)}{\partial x} \cdot \frac{\Phi(x)}{\phi(x)}[\phi(x) \mathrm{d} x .\right. \\
& =\int_{a}^{b}
\end{aligned}
$$

Therefore, the government's expected utility function can be rewritten as

$$
\begin{aligned}
\mathrm{E}[V(q(\xi), t(\xi), \xi)]= & \int_{a}^{b} \lambda G(q(x)) \phi(x) \mathrm{d} x-\int_{a}^{b} \lambda C(q(x), x) \phi(x) \mathrm{d} x \\
& +\int_{a}^{b}(1-\lambda)\left(p_{0}-p(x)\right) \phi(x) \mathrm{d} x \\
& -\int_{a}^{b} \lambda \Phi(x) \frac{\partial C(q(x), x)}{\partial x} \mathrm{~d} x
\end{aligned}
$$

Let

$$
F(q, x)=\lambda\left(G(q(x))-C(q(x), x)-\frac{\partial C(q(x), x)}{\partial x} \cdot \frac{\Phi(x)}{\phi(x)}\right)+(1-\lambda)\left(p_{0}-p(x)\right) .
$$


By taking the first-order and second-order partial derivative of $F(q, x)$ with respect to $x$, we obtain

$$
\begin{gathered}
\frac{\partial F(q, x)}{\partial q}=\lambda\left[\frac{\mathrm{d} G(q(x))}{\mathrm{d} q}-\frac{\partial C(q(x), x)}{\partial q}-\frac{\partial^{2} C(q(x), x)}{\partial x \partial q} \cdot \frac{\Phi(x)}{\phi(x)}\right] \\
\frac{\partial^{2} F(q, x)}{\partial q^{2}}=\lambda\left[\frac{\mathrm{d}^{2} G(q(x))}{\mathrm{d} q^{2}}-\frac{\partial^{2} C(q(x), x)}{\partial q^{2}}-\frac{\partial^{3} C(q(x), x)}{\partial x \partial q^{2}} \cdot \frac{\Phi(x)}{\phi(x)}\right] .
\end{gathered}
$$

According to assumptions (3.1) and (3.4), it is apparent that $\left(\partial^{2} F(q, x) / \partial q^{2}\right) \leq 0$; that is, the government's utility function $V(q, t, x)$ is concave in $q$. Therefore, the optimal output $q^{*}(x)$ satisfies $\left.(\partial F(q, x) / \partial q)\right|_{q=q^{*}(x)}=0$; that is,

$$
\frac{\mathrm{d} G\left(q^{*}(x)\right)}{\mathrm{d} q}-\frac{\partial C\left(q^{*}(x), x\right)}{\partial q}-\frac{\partial^{2} C\left(q^{*}(x), x\right)}{\partial x \partial q} \cdot \frac{\Phi(x)}{\phi(x)}=0 .
$$

Under such settings, the optimal transfer payment $t^{*}(x)$ can be written as

$$
t^{*}(x)=\beta x+C\left(q^{*}(x), x\right)+\int_{x}^{b} \frac{\partial C\left(q^{*}(\tau), \tau\right)}{\partial \tau} \mathrm{d} \tau .
$$
to $x$ is

In addition, the derivation of equation (42) with respect

$$
\begin{aligned}
& \left(\frac{\mathrm{d}^{2} G\left(q^{*}(x)\right)}{\mathrm{d} q^{2}}-\frac{\partial^{2} C\left(q^{*}(x), x\right)}{\partial q^{2}}-L(x) \frac{\partial^{3} C\left(q^{*}(x), x\right)}{\partial x \partial q^{2}}\right) \frac{\mathrm{d} q^{*}(x)}{\mathrm{d} x} \\
& =\frac{\partial^{2} C\left(q^{*}(x), x\right)}{\partial q \partial x}+\frac{\partial^{2} C\left(q^{*}(x), x\right)}{\partial q \partial x} \cdot \frac{\mathrm{d} L(x)}{\mathrm{d} x}+L(x) \frac{\partial^{3} C\left(q^{*}(x), x\right)}{\partial q \partial x^{2}},
\end{aligned}
$$

where $L(x)=(\Phi(x) / \phi(x))$. From assumptions (3.1) and (3.4), we have $\left(\mathrm{d} q^{*}(x) / \mathrm{d} x\right)<0$. Hence, the feasible solution $\left(q^{*}(x), t^{*}(x)\right)$ is the optimal solution of model (30).

By using Theorem 1, we can convert the uncertain pollution abatement problem (11) into an optimal control problem with boundary constraints by substituting equation (34) into the government's objective function. Theorem 1 designs the optimal contract $\left(q^{*}(x), t^{*}(x)\right)$ for the uncertain pollution abatement problem. This implies that the supply chain enterprise has the optimal output and the government pays the enterprise the optimal transfer payment. The incentive contract $\left(q^{*}(x), t^{*}(x)\right)$ is also the optimal mechanism designed by the government.

Let $u(x)=\mathrm{d} q(x) / \mathrm{d} x$. By converting the proposed model into the following optimal control problem, we obtain the necessary conditions for the optimal solution by applying Pontryagin's maximum principle.

Remark 1. The uncertain pollution abatement problem (11) can be cast as the following optimal control problem:

$$
\begin{array}{ll} 
& \max _{u(\cdot)}^{b} \lambda\left(G(q(x))-C(q(x), x)-\frac{\partial C(q(x), x)}{\partial x} \cdot \frac{\Phi(x)}{\phi(x)}\right) \phi(x) \mathrm{d} x \\
& +\int_{a}^{b}(1-\lambda)\left(p_{0}-p(x)\right) \phi(x) \mathrm{d} x \\
& \frac{\mathrm{d} q(x)}{\mathrm{d} x}=u(x), \quad \forall x \in[a, b] \\
\text { s.t. } \quad u(x)<0, \quad \forall x \in[a, b], \\
& t(b)-\beta b-C(q(b), b)=0 .
\end{array}
$$


In fact, construct the Hamiltonian $H(q(x), u(x)$, $\gamma(x), x)$ as

$$
\begin{aligned}
H(q(x), u(x), \gamma(x), x)= & \lambda\left(G(q(x))-C(q(x), x)-\frac{\partial C(q(x), x)}{\partial x} \frac{\Phi(x)}{\phi(x)}\right) \phi(x) \\
& +(1-\lambda)\left(p_{0}-p(x)\right) \phi(x)+\gamma(x) u(x),
\end{aligned}
$$

where $q(x)$ is the state variable, $u(x)$ is the control variable, and $\gamma(x)$ is the adjoint variable. From Pontryagin's maximum principle, the necessary condition of the optimal solution to model (45) is that there exists an adjoint variable $\gamma^{*}(\cdot)$ such that $\left(q^{*}(\cdot), u^{*}(\cdot)\right)$ satisfies the following:

(1) $u^{*}(\cdot)$ maximizes the Hamiltonian function (46); that is,

$$
H\left(q^{*}(x), u^{*}(x), \gamma^{*}(x), x\right)=\max _{u(x) \leq 0} H\left(q^{*}(x), u(x), \gamma^{*}(x), x\right) .
$$

(2) $q^{*}(x)$ satisfies the state equation

$$
\frac{\mathrm{d} q(x)}{\mathrm{d} x}=\frac{\partial H}{\partial \gamma} \text {. }
$$

(3) $\gamma^{*}(x)$ satisfies the adjoint equation

$$
\frac{\mathrm{d} \gamma(x)}{\mathrm{d} x}=-\frac{\partial H}{\partial q} \text {. }
$$

(4) The terminal constraints are satisfied; that is,

$$
\left\{\begin{array}{l}
\gamma(a)=0, \\
t(b)-\beta b-C(q(b), b)=0 .
\end{array}\right.
$$

From the above statement, it can be seen from equations (31) and (32) that the optimal contract $\left(q^{*}(x), t^{*}(x)\right)$ can also be obtained by applying Pontryagin's maximum principle. Furthermore, we investigate the effects of carbon emission level on the supply chain enterprise's output and the government's transfer payment in the following proposition.

Proposition 4. The optimal contract $\left(q^{*}(x), t^{*}(x)\right)$ for model (30) has the following features:

(i) $q^{*}(x)$ is decreasing in $x$.

(ii) If there exist a tax rate $\beta$ and a carbon emission level $x$ such that $\beta+\left(\partial C\left(q^{*}(x), x\right) / \partial q^{*}(x)\right) \quad\left(\partial q^{*}(x) /\right.$ $\partial x) \leq 0$, then $t^{*}(x)$ is decreasing in $x$; if there also exist $a$ tax rate $\beta$ and a carbon emission level $x$ such that $\beta+\left(\partial C\left(q^{*}(x), x\right) / \partial q^{*}(x)\right)\left(\partial q^{*}(x) / \partial x\right)>0, \quad$ then $t^{*}(x)$ is increasing in $x$.
Proof. Result (i) can be obtained immediately from the proof of Theorem 1.

For result (ii), due to $\mathrm{d} q^{*}(x) / \mathrm{d} x<0$, $\partial C\left(q^{*}(x), x\right) / \partial q^{*}(x)>0$ and

$$
\frac{\mathrm{d} t^{*}(x)}{\mathrm{d} x}=\beta+\frac{\partial C\left(q^{*}(x), x\right)}{\partial q^{*}(x)} \cdot \frac{\mathrm{d} q^{*}(x)}{\mathrm{d} x},
$$

and it is clear that $\mathrm{d} t^{*}(x) / \mathrm{d} x<0$ on the condition that $0<\beta \leq-\left(\partial C\left(q^{*}(x), x\right) / \partial q^{*}(x)\right)\left(\partial q^{*}(x) / \partial x\right)$. Moreover, it is also obtained that $\mathrm{d} t^{*}(x) / \mathrm{d} x>0$ on the condition that $-\left(\partial C\left(q^{*}(x), x\right) / \partial q^{*}(x)\right)\left(\partial q^{*}(x) / \partial x\right)<\beta<1$.

Proposition 4 implies that as the realized carbon emission level increases, the supply chain enterprise lowers the output to reduce the pollution emissions. We assumed that the supply chain enterprise's production cost increases with the realized carbon emission level. In this assumption, it is believed that the increase in production cost may result in a reduction in the supply chain enterprise's output. Moreover, an increase in the carbon emission level will also affect the government's transfer payment to the supply chain enterprise. If the tax rate of the carbon emissions quota is below a certain threshold $\left(0<\beta \leq-\left(\partial C\left(q^{*}(x), x\right) / \partial q^{*}(x)\right)\right.$ $\left.\left(\partial q^{*}(x) / \partial x\right)\right)$, the government's transfer payment will decrease with the carbon emission level to reduce the pollution emissions. However, if the tax rate of this quota is above this threshold $\quad\left(-\left(\partial C\left(q^{*}(x), x\right) / \partial q^{*}(x)\right)\left(\partial q^{*}(x) / \partial x\right)<\beta<1\right)$, the government's transfer payment to the regulated polluting supply chain enterprise will increase with the carbon emission level. This finding leads us to an interesting conclusion that the impacts of the government's transfer payment on carbon emission level have opposite effects under different tax rates. Specifically, an increase in carbon emissions could increase the government's transfer payment given a high tax rate for the carbon emissions quota. When the carbon emission level increases, so do the supply chain enterprise's production cost and carbon tax. Moreover, the regulated polluting supply chain enterprise's utility decreases with the increase in production cost and carbon tax. Then, the government's transfer payment to the supply chain enterprise may increase. The reason is that the government should encourage the supply chain enterprise to participate in production. Furthermore, it also follows from the participation constraint that when the carbon emission level increases further, the incentive for the government to increase the transfer payment becomes stronger. 
Additionally, the government collects a carbon tax from the supply chain enterprise. The carbon tax consists of the tax rate of the carbon emission quota and the carbon emission level. Therefore, as the tax rate or the carbon emission level increases, so will the carbon tax, and then the government's transfer payment will decrease. In reality, it is believed that the government often wants to use a carbon tax to control the carbon emission level and improve the utility. According to Proposition 4, a decrease in the carbon emission level will increase the supply chain enterprise's output. However, a decrease in the carbon emission level would decrease the government's transfer payment if the carbon tax is high. The following proposition answers the question of how carbon emissions affect the government and the supply chain enterprise's optimal utilities.
Proposition 5. The optimal utilities $V\left(q^{*}(x), t^{*}(x), x\right)$ and $U\left(q^{*}(x), t^{*}(x), x\right)$ of the uncertain pollution abatement model satisfy the following:

(i) The government's optimal utility $V\left(q^{*}(x), t^{*}(x), x\right)$ is decreasing in the carbon emission level $x$.

(ii) The supply chain enterprise's optimal utility $U\left(q^{*}(x), t^{*}(x), x\right)$ is decreasing in the carbon emission level $x$, and his marginal utility is also increasing in the carbon emission level $x$.

Proof. For result (i), by taking the partial derivative of equation (7) with $x$, we have

$$
\frac{\partial V\left(q^{*}(x), t^{*}(x), x\right)}{\partial x}=\lambda\left(\frac{\partial G\left(q^{*}(x)\right)}{\partial q^{*}(x)} \frac{\partial q^{*}(x)}{\partial x}-\frac{\partial t^{*}(x)}{\partial x}+\beta\right)-(1-\lambda) \frac{\partial p(x)}{\partial x} .
$$

According to Proposition 1, it is clear that

$$
\begin{aligned}
\frac{\partial V\left(q^{*}(x), t^{*}(x), x\right)}{\partial x} & =\lambda\left(\frac{\partial G\left(q^{*}(x)\right)}{\partial q^{*}(x)} \frac{\partial q^{*}(x)}{\partial x}-\frac{\partial C\left(q^{*}(x), x\right)}{\partial q^{*}(x)} \cdot \frac{\partial q^{*}(x)}{\partial x}\right)-(1-\lambda) \frac{\partial p(x)}{\partial x} \\
& =\lambda\left(\frac{\partial G\left(q^{*}(x)\right)}{\partial q^{*}(x)}-\frac{\partial C\left(q^{*}(x), x\right)}{\partial q^{*}(x)}\right) \frac{\partial q^{*}(x)}{\partial x}-(1-\lambda) \frac{\partial p(x)}{\partial x} .
\end{aligned}
$$

From assumptions (3.2) and (3.5), we have $(\partial p(x) / \partial x)>0$ and $\left(\partial G\left(q^{*}(x)\right) / \partial q^{*}(x)\right)-\left(\partial C\left(q^{*}(x)\right.\right.$, $\left.x) / \partial q^{*}(x)\right)>0$.

Furthermore, it follows from Proposition 1 that

$$
\frac{\partial V\left(q^{*}(x), t^{*}(x), x\right)}{\partial x}<0 .
$$

For result (ii), by taking the partial derivative of equation (10) with respect to $x$, we also have

$$
\frac{\partial U\left(q^{*}(x), t^{*}(x), x\right)}{\partial x}=\frac{\partial t^{*}(x)}{\partial x}-\beta-\frac{\partial C\left(q^{*}(x), x\right)}{\partial x}-\frac{\partial C\left(q^{*}(x), x\right)}{\partial q^{*}(x)} \frac{\partial q^{*}(x)}{\partial x} .
$$

From Proposition 1, we obtain that

$$
\frac{\partial U\left(q^{*}(x), t^{*}(x), x\right)}{\partial x}=-\frac{\partial C\left(q^{*}(x), x\right)}{\partial x}<0 .
$$

Furthermore,

$$
\frac{\partial^{2} U\left(q^{*}(x), t^{*}(x), x\right)}{\partial^{2} x}=-\frac{\partial^{2} C\left(q^{*}(x), x\right)}{\partial x \partial q^{*}(x)} \frac{\partial q^{*}(x)}{\partial x} .
$$

From assumption (3.1) and Proposition 1, it is apparent that

$$
\begin{aligned}
\frac{\partial^{2} C\left(q^{*}(x), x\right)}{\partial x \partial q^{*}(x)} & >0, \\
\frac{\partial q^{*}(x)}{\partial x} & <0 .
\end{aligned}
$$

Therefore, we have

$$
\frac{\partial^{2} U\left(q^{*}(x), t^{*}(x), x\right)}{\partial^{2} x}>0
$$

This proposition indicates that the government and the supply chain enterprise's optimal utilities will decrease as the carbon emission level increases. Previously, we determined that an increase in carbon emissions increased the government's transfer payment when the carbon tax was high. Although the transfer payment increases, the carbon tax $\beta x$ also increases and then negatively affects the utility of the supply chain enterprise. Therefore, the above conclusion is reasonable. If the carbon $\operatorname{tax} \beta x$ is constant, the carbon emission level $x$ decreases with the increase in tax rate $\beta$. Therefore, the tax rate could control the carbon emission 
level. When holding the carbon emission level $x$ constant, an increase in the tax rate will lead to deterioration of the supply chain enterprise's utility. To maintain the participation constraint, the supply chain enterprise would lower the production cost. Based on assumption (3.1), it is observed that the production cost $C(q(x), x)$ decreases as the output $q(x)$ decreases. Thus, an increase in the tax rate will reduce the regulated polluting supply chain enterprise's optimal output.

\section{Numerical Example}

In this section, we provide a numerical example to illustrate the effectiveness of the optimal contract and investigate the effects of carbon emissions, carbon tax rate, and environmental preference on the optimal contract design. Without loss of generality, we assume that the supply chain enterprise's carbon emission level $\xi=\mathscr{L}(a, b)$ is a linear uncertain variable; that is, the supply chain enterprise's carbon emission level has the minimum value $a$ and the maximum value $b$.

Assume that the government's revenue function is $G(q(x))=\ln q(x)$, and the supply chain enterprise's production cost is $C(q, x)=x^{2} q$. The degree of pollution to the environment is $p(x)=e^{x}$, and $p_{0}=e^{b}$. Therefore, the government's utility function can be written as

$$
V(q(x), t(x), x)=\lambda(\ln q(x)-t(x)+\beta x)+(1-\lambda)\left(e^{b}-e^{x}\right), \quad x \in[a, b]
$$

The supply chain enterprise's utility function can be written as

$$
U(q(x), t(x), x)=t(x)-\beta x-x^{2} q(x), \quad x \in[a, b] .
$$

The uncertain pollution abatement model is

$$
\begin{array}{ll}
\max _{\{q(\cdot), t(\cdot)\}} E\left[\lambda(\ln q(x)-t(x)+\beta x)+(1-\lambda)\left(e^{b}-e^{x}\right)\right] \\
& t(x)-\beta x-x^{2} q(x) \geq t(y)-\beta y-x^{2} q(y), \quad \forall x, y \in[a, b], \\
\text { s.t. } \quad & t(x)-\beta x-x^{2} q(x) \geq 0, \quad \forall x \in[a, b] .
\end{array}
$$

According to Propositions 1-3, it follows from equation (30) that model (62) can be described as

$$
\begin{aligned}
& \max _{\{q(\cdot), t(\cdot)\}} \int_{a}^{b}\left[\lambda(\ln q(x)-t(x)+\beta x)+(1-\lambda)\left(e^{b}-e^{x}\right)\right] \phi(x) \mathrm{d} x \\
& \qquad \frac{\mathrm{d} t(x)}{\mathrm{d} x}=\beta+x^{2} \frac{\mathrm{d} q(x)}{\mathrm{d} x}, \quad \forall x \in[a, b], \\
& \text { s.t. } \quad \frac{\mathrm{d} q(x)}{\mathrm{d} x}<0, \quad \forall x \in[a, b], \\
& t(b)-\beta b-b^{2} q(b)=0 .
\end{aligned}
$$

From equations (31) and (32) in Theorem 1, the optimal contract $\left(q^{*}(x), t^{*}(x)\right)$ to model (63) satisfies

$$
\begin{array}{r}
\frac{1}{q^{*}(x)}-x^{2}-2 x \frac{\Phi(x)}{\phi(x)}=0, \\
t^{*}(x)=\beta x+x^{2} q^{*}(x)+\int_{x}^{b} 2 \tau q^{*}(\tau) \mathrm{d} \tau .
\end{array}
$$

Since it is clear that

$$
\Phi(x)= \begin{cases}0, & \text { if } x<a, \\ \frac{x-a}{b-a}, & \text { if } a \leq x \leq b, \\ 1, & \text { if } x>b,\end{cases}
$$




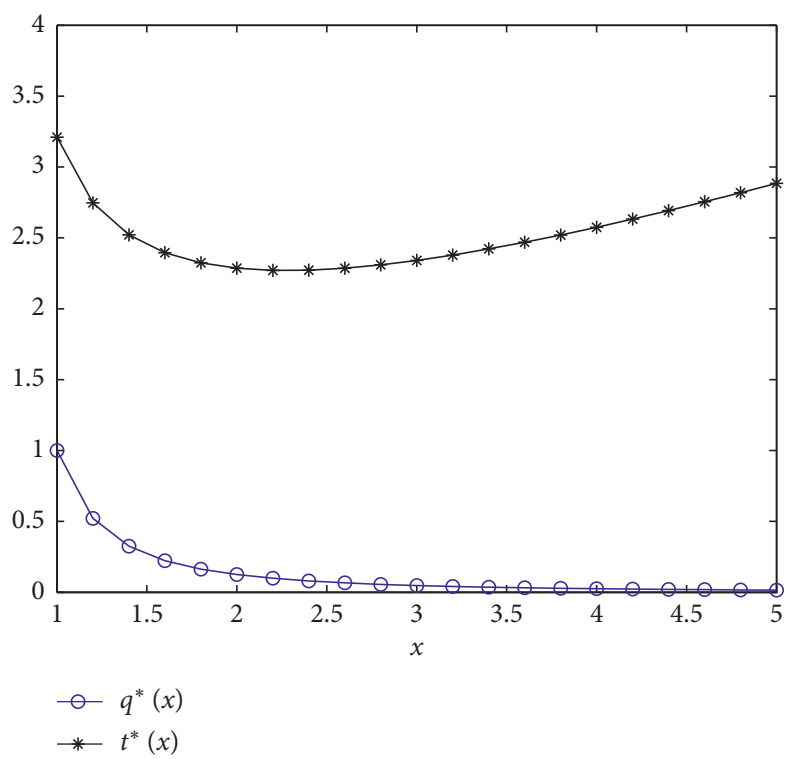

FIgURE 1: Optimal solutions of uncertain pollution abatement model.

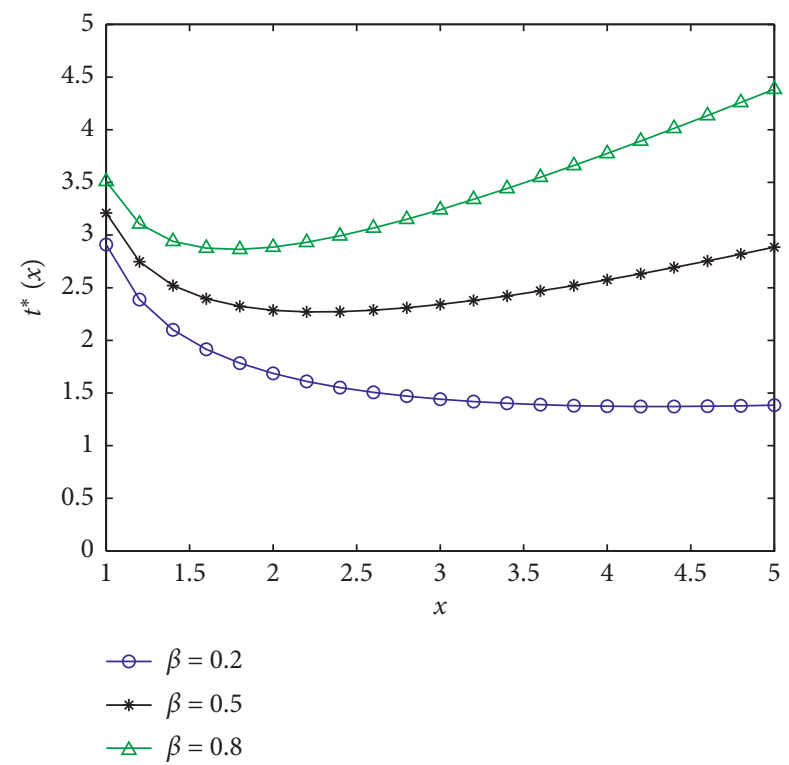

Figure 2: Effect of carbon tax rate on optimal transfer payment.

and $(\Phi(x) / \phi(x))=x-a, \forall x \in[a, b]$, we obtain that

$$
\begin{gathered}
q^{*}(x)=\frac{1}{3 x^{2}-2 a x}, \\
t^{*}(x)=\beta x+\frac{x}{3 x-2 a}+\frac{2}{3} \ln (3 b-2 a)-\frac{2}{3} \ln (3 x-2 a) .
\end{gathered}
$$

In particular, if $a=1, b=5$, and $\beta=0.5$, the optimal contract $\left(q^{*}(x), t^{*}(x)\right)$ obtained from equations $(66)$ and (67) is shown in Figure 1. It can be observed from Figure 1 that the optimal design of the pollution abatement contract generally involves a negative supply chain enterprise's output, and it would be the optimal strategy for the government to order a reduction in the supply chain enterprise's output when the supply chain enterprise's carbon emission level increases. Moreover, when the supply chain enterprise's carbon emission level is below a certain threshold, the government's transfer payment will decrease with the carbon emission level. When the supply chain enterprise's carbon emission level is above that threshold, the government's transfer payment will increase with the carbon emission level.

Furthermore, we examine the effect of the carbon tax rate on the government's optimal transfer payment. Without loss of generality, we assume that the carbon tax rate $\beta$ can be chosen from the set $\{0.2,0.5,0.8\}$ and that the parameters $a=1$ and $b=5$ remain unchanged. From Figure 2, we 


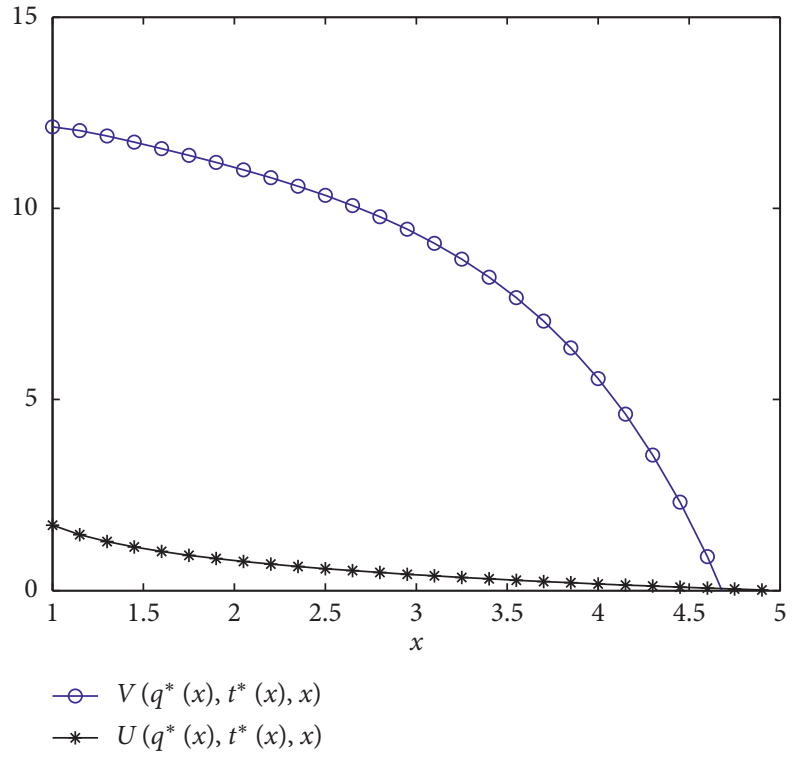

(a)

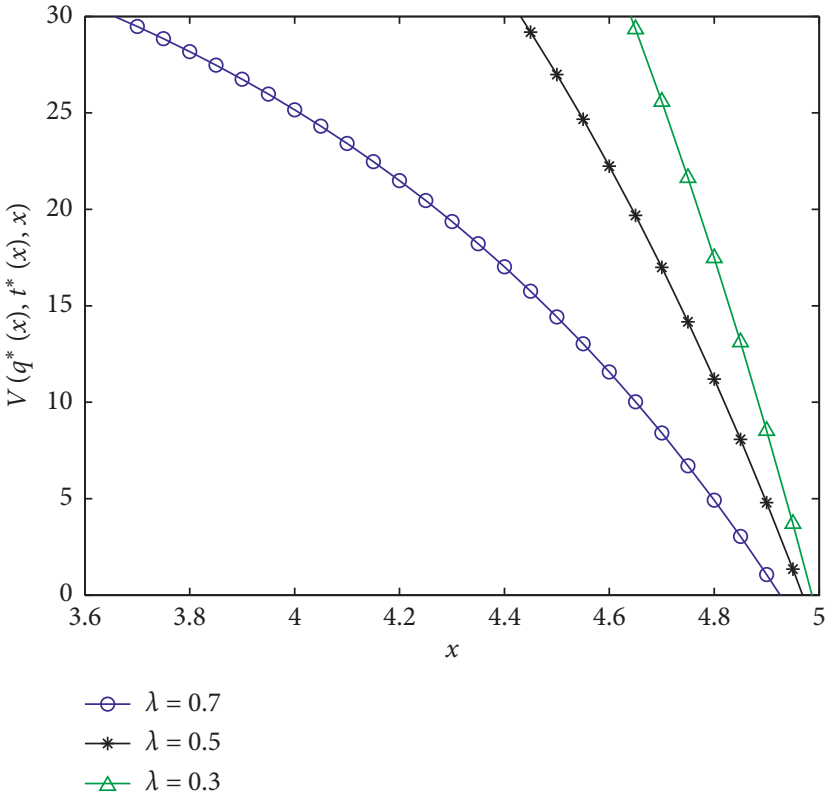

(b)

FIGURE 3: Optimal utility of uncertain pollution abatement model. (a) Effect of carbon emission on optimal utility. (b) Effect of environmental preference on government's utility.

observe that the government's transfer payment is decreasing in the carbon emission level if the carbon tax rate is low. When the carbon tax rate increases, the government's transfer payment shows a rising trend as the carbon emission level increases.

Finally, we illustrate the optimal utility of the uncertain pollution abatement model and examine the impacts of carbon emissions and environmental preference on the optimal utility. Suppose that the parameters $a=1, b=5$, and $\lambda=0.9$ remain unchanged. Figure 3(a) shows that the optimal utility of the uncertain pollution abatement model decreases with the carbon emission level. However, an increase in the carbon emission level will lead to the government's diminishing marginal utility and the supply chain enterprise's incremental marginal utility. To test and verify the effect of the environmental preference on the government's utility, the environmental preference $\lambda$ can be chosen from the set $\{0.7,0.5,0.3\}$. Accordingly, Figure $3(\mathrm{~b})$ shows that the government's utility will increase as the environmental preference decreases, that is, as the government becomes more concerned with the environment.

\section{Conclusion}

This paper studies an uncertain contract model for the pollution abatement problem in which the government faces the supply chain enterprise with private carbon emission information. The optimal contract model, in which the government's assessment of the supply chain enterprise's carbon emission level is characterized as an uncertain variable, is presented with the purpose of maximizing the expected utility of the government. The crisp equivalent model for the uncertain contract model is presented and the optimal solution of the equivalent model is obtained. The results show that the regulated polluting supply chain enterprise's optimal output will decrease if the supply chain enterprise's carbon emission level increases. Additionally, if the carbon tax is below a certain threshold, the government's optimal transfer payment decreases with the carbon emission level. However, we reach an interesting conclusion that the impacts of the government's transfer payment on carbon emission level have opposite effects under different carbon tax rates. The government's optimal transfer payment to the regulated polluting supply chain enterprise increases with the carbon emission level if the carbon tax is above a threshold. In addition, the government and the supply chain enterprise's optimal utilities will strictly decrease as the supply chain enterprise's carbon emission level increases.

In future work, we could incorporate competition into the contract design in the pollution abatement problem. For instance, the government may offer an incentive contract to motivate supply chain enterprises and induce competition among them. Furthermore, it would also be interesting to study the bounded rationality decision in the optimal incentive contracts for the pollution abatement problem.

\section{Data Availability}

The data of numerical example used to support the findings of this study are included within the article.

\section{Conflicts of Interest}

The authors declare that they have no conflicts of interest. 


\section{Acknowledgments}

This work was supported by Humanity and Social Science Youth Foundation of Ministry of Education of China (no. 16YJC630159), the National Natural Science Foundation of China (no. 71702129), and China Postdoctoral Science Foundation (no. 2017M6101 60).

\section{References}

[1] S. Pacala and R. Socolow, "Stabilization wedges: solving the climate problem for the next 50 years with current technologies," Science, vol. 305, no. 5686, pp. 968-972, 2004.

[2] R. Martin, L. B. de Preux, and U. J. Wagner, "The impact of a carbon tax on manufacturing: evidence from microdata," Journal of Public Economics, vol. 117, no. 1, pp. 1-14, 2014.

[3] J.-C. Wagner and K.-B. Chang, "An optimal tax/subsidy for output and pollution control under asymmetric information in oligopoly markets," Journal of Regulatory Economics, vol. 5, no. 2, pp. 183-197, 1993.

[4] J. Duggan and J. Roberts, "Implementing the efficient allocation of pollution," American Economic Review, vol. 92, no. 4, pp. 1070-1078, 2002.

[5] J.-P. Montero, "A simple auction mechanism for the optimal allocation of the commons," American Economic Review, vol. 98, no. 1, pp. 496-518, 2008.

[6] X. Liu, C. Wang, D. Niu, S. Suk, and C. Bao, "An analysis of company choice preference to carbon tax policy in China," Journal of Cleaner Production, vol. 103, no. 1, pp. 393-400, 2015.

[7] M. Wang, M. Wang, C. Dang, and S. Wang, "A pareto optimal auction mechanism for carbon emission rights," Mathematical Problems in Engineering, vol. 2014, Article ID 438104, 7 pages, 2014.

[8] Y. Li, Q. Deng, C. Zhou, and L. Feng, "Environmental governance strategies in a two-echelon supply chain with tax and subsidy interactions," Annals of Operations Research, vol. 290, no. 1-2, pp. 439-462, 2020.

[9] J.-J. Laffont and J. Tirole, "Pollution permits and compliance strategies," Journal of Public Economics, vol. 62, no. 1-2, pp. 85-125, 1996.

[10] S. Lothe and I. Myrtveit, "Compensation systems for green strategy implementation: parametric and non-parametric approaches," Business Strategy and the Environment, vol. 12, no. 3, pp. 191-203, 2003.

[11] B. Fahimnia, J. Sarkis, A. Choudhary, and A. Eshragh, "Tactical supply chain planning under a carbon tax policy scheme: a case study," International Journal of Production Economics, vol. 164, no. 1, pp. 206-215, 2015.

[12] D. Klenert and L. Mattauch, "How to make a carbon tax reform progressive: the role of subsistence consumption," Economics Letters, vol. 138, no. 1, pp. 100-103, 2016.

[13] X. Zhao, Y. Li, F. Xu, and K. Dong, "Sustainable collaborative marketing governance mechanism for remanufactured products with extended producer responsibility," Journal of Cleaner Production, vol. 166, no. 1, pp. 1020-1030, 2017.

[14] C. Helm and F. Wirl, "The principal-agent model with multilateral externalities: an application to climate agreements," Journal of Environmental Economics and Management, vol. 67, no. 1, pp. 141-154, 2014.

[15] C. Arguedas and S. Rousseau, "Emission standards and monitoring strategies in a hierarchical setting," Environmental and Resource Economics, vol. 60, no. 3, pp. 395-412, 2015.
[16] R. K. Shrestha, "Menus of price-quantity contracts for inducing the truth in environmental regulation," Journal of Environmental Economics and Management, vol. 83, no. 1, pp. 1-7, 2017.

[17] A. Lika, F. Galioto, and D. Viaggi, "Water authorities' pricing strategies to recover supply costs in the absence of water metering for irrigated agriculture," Sustainability, vol. 9, no. 12, pp. 1-16, 2017.

[18] R. Dubey, A. Gunasekaran, P. Helo, T. Papadopoulos, S. J. Childe, and B. S. Sahay, "Explaining the impact of reconfigurable manufacturing systems on environmental performance: the role of top management and organizational culture," Journal of Cleaner Production, vol. 141, no. 1, pp. 56-66, 2017.

[19] Q. Song and K. Shi, "A fuzzy waiting time contract for patient's public health care," Journal of Intelligent \& Fuzzy Systems, vol. 27, no. 2, pp. 1001-1009, 2014.

[20] H. Zhang and J. Jiang, "Informed principal model and contract in supply chain with demand disruption asymmetric information," Mathematical Problems in Engineering, vol. 2016, Article ID 2306583, 12 pages, 2016.

[21] B. Liu, Uncertainty Theory, Springer, Berlin, Germany, 2nd edition, 2007.

[22] Z. Chen, Y. Lan, and R. Zhao, "Impacts of risk attitude and outside option on compensation contracts under different information structures," Fuzzy Optimization and Decision Making, vol. 17, no. 1, pp. 13-47, 2018.

[23] R. Mu, Y. Lan, and W. Tang, "An uncertain contract model for rural migrant worker's employment problems," Fuzzy Optimization and Decision Making, vol. 12, no. 1, pp. 29-39, 2013.

[24] C. Zhou, J. Peng, Z. Liu, and B. Dong, "Optimal incentive contracts under loss aversion and inequity aversion," Fuzzy Optimization and Decision Making, vol. 18, no. 1, pp. 85-102, 2019.

[25] C. Zhou, G. Xu, and Z. Liu, "Incentive contract design for internet referral services: cost per click vs cost per sale," Kybernetes, vol. 49, no. 2, pp. 601-626, 2020.

[26] C. Zhou, N. Ma, X. Cui, and Z. Liu, "The impact of online referral on brand market strategies with consumer search and spillover effect," Soft Computing, vol. 24, no. 4, pp. 2551-2565, 2020.

[27] Z. Liu, C. Zhou, H. Chen, and R. Zhao, "Impact of cost uncertainty on supply chain competition under different confidence levels," International Transactions in Operational Research, vol. 28, no. 3, pp. 1465-1504, 2021.

[28] Y. Liu and M. Ha, "Expected value of function of uncertain variables," Journal of Uncertain Systems, vol. 4, no. 3, pp. 181-186, 2010. 